\title{
Simple-Graphs Fusion in Image Mosaic: Application to Automated Cell Files Identification in Wood Slices
}

\author{
Guilhem Brunel $^{1,2, *}$, Philippe Borianne ${ }^{1}$, Gérard Subsol ${ }^{3}$, and Marc Jaeger ${ }^{1}$ \\ ${ }^{1}$ CIRAD - UMR AMAP, France \\ ${ }^{2}$ Université Montpellier 2, France \\ ${ }^{3}$ CNRS - LIRMM, France \\ guilhem.brunel@cirad.fr
}

\begin{abstract}
Results aggregation by disjoint graph merging is potentially a good alternative to image stitching. During the processing of image mosaics, it allows to be free of radiometric and geometric corrections inherent in image fusion. We have studied and developed a generic merging method of disjoint graphs for tracking cell alignments in image mosaics of wood.
\end{abstract}

Keywords: graphs theory, graphs fusion, image processing, pattern recognition, cell segmentation, cell organization.

\section{$1 \quad$ Introduction}

Graph theory is widely used in image processing [1] especially for region encoding or image segmentation. The image is then considered as a graph whose vertex set is made of pixels, and edge set is given by an adjacency relation between them. Numerous segmentation methods based on region merging are proposed in literature, the most known being the Split and Merge algorithm. Several authors combine it with the watershed algorithm by using graph fusion to segment grey [2] or color [3-4] images. In all these approaches, the fusion operates on the vertices of a single graph.

Our specific segmentation method of cell alignments, so called cell files, in wood slices produces an adjacency graph. Nevertheless, identifying cell files on very large areas given by images mosaic implies either to process the image resulting from combining all the images of the mosaic or to merge the results produced by processing each image.

In the first case, stitching is the method which is the most often used. Image stitching combines multiple images with overlapping fields of view in order to produce a high-resolution image. Most of the algorithms require nearly exact overlaps between images and identical exposures to produce seamless results [5-6]. Moreover, they often request local deformation correction [7-9] due to optical acquisition properties, or local offset rectification [10] due to the automated views shooting. However, the resulting high resolution images may still be difficult to be processed, especially from the final resolution (up to several billion pixels) and the possible radiative variation due to the microscope acquisition properties.

\footnotetext{
* Corresponding author.
} 
So, we preferred to use the second class of methods. Our framework is based on the understanding of the cambium growth mechanisms which requires the studies of cell patterns regularity, of their disruption or of their modification through space and time [11-12]. We are interesting by radial files (cell alignments); they help understanding the development, the differentiation and the temporal changes of cells [13]. The automation of the cell files identification and the characterization of their components [14-15] allow today to process a large quantity of images. However, we aim to study a large area of observation, following cells of some microns diameter on a given wood slice of several centimeters diameter. But these recognition processes being constrained to single image detections; studies of large area are thus impracticable. Identifying radial files on large areas described by images mosaic involves therefore merging the results produced by the processing of each image.

Our dataset is defined by color images mosaic of wood histological slices. Transversal slices of $20 \mu \mathrm{m}$ thickness are prepared with a vibratome, colored, and then fit in between glass strips before being digitalized by a camera coupled with the microscope. The mosaic images (Fig. 1.) are successively generated by progressive displacement of the microscope stage taking the strip. The position of the image in the mosaic is given by the stage displacement in abscissa $\mathrm{X}$ and ordinate $\mathrm{Y}$. The observation area is limited to $10 \times 10 \mathrm{~cm}^{2}$, requiring nearly 1500 images mosaic. Each image is encoded in RGB system, with a resolution of 1600 by 1200 pixels. The images may show local or peripheral blur due to the optical deformations or specimen thickness variations.

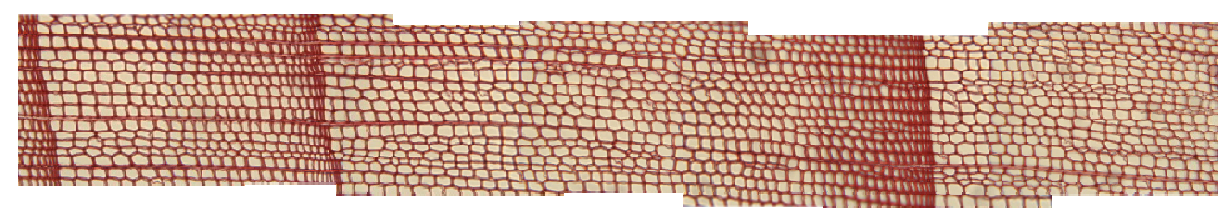

Fig. 1. Seven color images mosaic of Black Pinus overlapping a $2 \times 0.25 \mathrm{~cm}^{2}$ area

We wish to avoid images stitching difficulties related to the high resolution and the radiative corrections. We propose an approach mixing gradually the processing of images and the progressive merging of the produced results [16-17]. The merging process takes benefits from the result description using a graph. Such a model is well adapted to cells organization. The results merging of two adjacent images consist thus of their two respective graphs amalgamation.

The paper extends a preliminary work published in Fourth International Symposium on Plant Growth Modeling [14] presenting radial files identification in wood slice image. The following section describes the notion of simple-graph. Section 3 reminds the automated cell files identification principles in the microscopic images. Section 4 presents the graph merging and the fusion kernel notion. Results and perspectives are presented and discussed in the section 5 before conclusion. 
Our contribution lies in the graphs fusion formalism and its application:

- We introduce the Simple Graph notion to describe cell organization and the fusion kernel used to amalgamate two graphs;

- We introduce a similarity function in order to define the fusion kernel, i.e. the vertices of the graphs which should be amalgamated.

- We introduce a local blur indicator to define the best representative vertex in vertices fusion.

\section{Graph Theory Notion}

This section gives some general definitions about graphs merging mechanism. The only considered specificity is the nature of the graph, linked to the properties of cell files. We present here the formalization of the graphs amalgamation, i.e. when two distinct graphs are gathered by merging of one of their vertices.

\subsection{Simple Graph or S-Graph}

A graph $G=(V, E)$ is a mathematical structure composed of two finite sets $V$ and $E$. The elements of $\mathrm{V}$ are called vertices, and the elements of $\mathrm{E}$ are called edges. A simple graph or S-Graph is an undirected graph that has no loops and no more than one edge between any two different vertices. In a simple graph, the edges of the graph form a set and each edge is a distinct pair of vertices. In a simple graph of $\mathrm{n}$ vertices, every vertex has a degree that is less than $n$ (the converse, is not true - there exist non-simple graphs with $n$ vertices in which every vertex has a degree smaller than $n$ ).
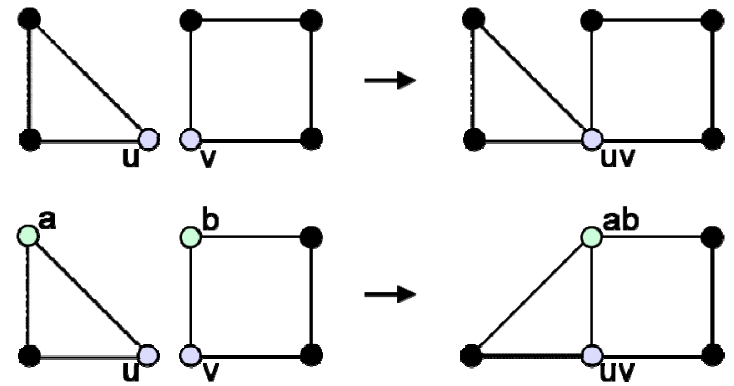

Fig. 2. Top, simple fusion of two nodes of disjoint S-graphs. Bottom, complex fusion of four nodes and two edges of disjoint S-graphs.

\subsection{Multiple S-Graph Amalgamation}

Let $G_{\mathrm{i}}=\left(V_{\mathrm{i}}, E_{\mathrm{i}}\right)$ and $G_{\mathrm{j}}=\left(V_{\mathrm{j}}, E_{\mathrm{j}}\right)$ be two disjoint S-graphs, with $K_{\mathrm{i}}=\left(A_{\mathrm{i}}, B_{\mathrm{i}}\right)$ a sub S-graph of $G_{\mathrm{i}}$ and $K_{\mathrm{j}}=\left(A_{\mathrm{j}}, B_{\mathrm{j}}\right)$ a sub S-graph of $G_{\mathrm{j}}$. Let $f: K_{\mathrm{i}} \rightarrow K_{\mathrm{j}}$ be an involution without fixed point between these subgraphs.

$$
\forall u \in K_{i} \quad f(u) \in K_{j} \text { and } f(f(u))=u \text { with } f(u) \neq u
$$


The amalgamation of $G_{\mathrm{i}}$ and $G_{\mathrm{j}}$ modulo the isomorphism $f$ is the S-graph obtained from the union $G_{\mathrm{i}} \cup G_{\mathrm{j}}$ by merging each vertex $a$ and each edge $b$ in the subgraph $K_{\mathrm{i}}$ with their respective images $f(a)$ and $f(b)$ in subgraph $K_{\mathrm{j}}$. The amalgamated vertex is noted $a f(a)$, and the amalgamated edge is noted $b f(b)$.

The vertex set of this new graph is $\left(V_{i}-A_{i}\right) \cup\left(V_{j}-B_{j}\right) \cup\left\{a f(a) \mid a \in A_{i}\right\}$, and the edge set is $\left(E_{i}-B_{i}\right) \cup\left(E_{j}-B_{j}\right) \cup\left\{e f(e) \mid e \in B_{i}\right\}$; any edge which had $a \in A_{i}$ or $f(a) \in A_{j}$ as endpoint now has the amalgamated vertex $a f(a)$ as endpoint. The amalgamation result is a $\mathrm{S}$-graph: each edge appears one and only time in the edges set as shown in Fig. 2.

\subsection{Amalgamation Kernel}

The amalgamation kernel is the set of the merged vertex couples defined as

$$
U V=\left\{u v \mid u \in A_{i} \text { and } v \in A_{j} \text { and } v=f(u)\right\}
$$

\section{The Cell File Identification Principle}

The cell files identification process [14] leads on the following principles: (i) the files are alignment of two by two similar cells, i.e. close in terms of size, shape and dynamic; (ii) the cells alignment are independent of the image orientation: the method have to be reversible.

From methodological point of view, the contrast between cell wall and lumen is enhanced by a difference of Gaussians on the color image (Fig. 3.). Then, the image is converted in grey levels by the simple mean of the color channels.
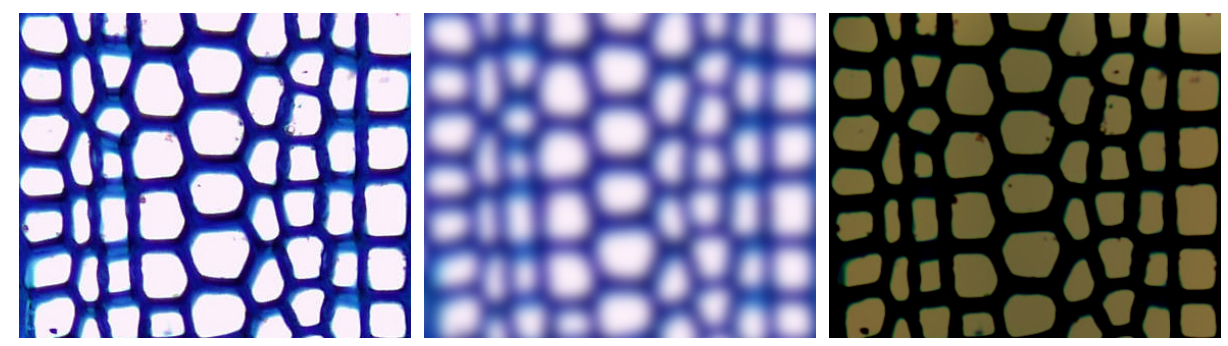

Fig. 3. Image contrast enhancing by a Difference of Gaussian filtering on color images of Pinus. Left: the image obtained by applying a small Gaussian blurring ( $\sigma=3$ pixels). Center: the image resulting from a strong Gaussian blurring $(\sigma=$ (image width)/10). Right: subtraction of previous images which increases the contrast between wall and lumen.

The cells individualization is built from watershed process [18]; the crest lines define regions whose limits correspond to the inter-cells separation (Fig. 4.). The cells adjacency graph is built from these lines [19]. The cell files are identified by reversible breadth-first search exploration of the graph under spatio-geometrical constraints: they are defined as the most linear paths whose adjacent vertices present similar characteristics, the vertex taking attributes describing shape, size and aspect of the cell. 

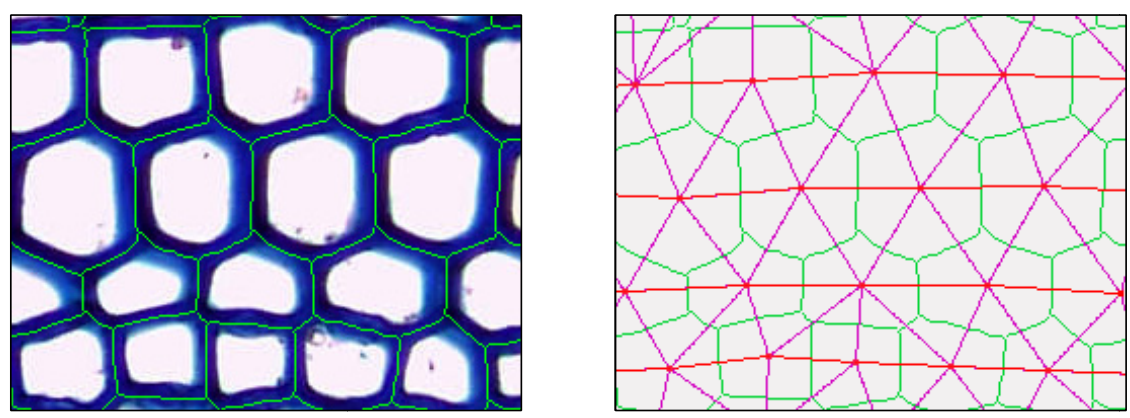

Fig. 4. Cells identification and its adjacency graph. Legend: in green, the crest lines; in red and pink, the adjacency graph; in red, the cell files. Left: the crest lines produced by the watershed algorithm define a good approximation of the cell contours. Right: the adjacency graph is built from the crest lines; the cell files are defined by the most linear paths of the graph.

\section{The Graphs Fusion Implementation}

This section specifies how the general definitions are implemented in our study context, in particular the way we define the amalgamation kernel.

Let $G=(V, E)$ be a given simple graph. At each vertex $v$ of $V$ is associated a set of spatio-geometrical attributes describing the cell c represented by $v$ :

$-r_{v}$, the radius of the circumscribed circle of $c$;

$-x_{v}$, the cell geometrical centre abscissa;

$-y_{v}$, the cell geometrical centre ordinate;

$-s_{v}$, the cell area.

\subsection{Similarity Coefficient}

The similarity coefficient estimates the likeness between vertices of distinct S-graphs. It is defined by a single product of attribute differences: each attribute has the same weight in the similarity evaluation. This coefficient tends toward 1 for similar vertices, towards 0 for too different or distant vertices.

Let $G_{0}=\left(V_{0}, E_{0}\right)$ and $G_{1}=\left(V_{1}, E_{1}\right)$ be two given S-graphs.

$\forall v \in G_{i}, \forall w \in G_{1-i}, i \in\{0,1\}$, the coefficient is defined by:

$$
\operatorname{sim}_{v, w} \begin{cases}0 & \text { if }|v, w|>\min \left(r_{v}, r_{w}\right) \\ \left(1-\frac{\left|x_{v}-x_{w}\right|}{x_{v}+x_{w}}\right)\left(1-\frac{\left|y_{v}-y_{w}\right|}{y_{v}+y_{w}}\right)\left(1-\frac{\left|S_{v}-S_{w}\right|}{S_{v}+S_{w}}\right) & \text { if }|v, w| \leq \min \left(r_{v}, r_{w}\right)\end{cases}
$$

This definition distinguishes the case of too distant vertices from the case of potentially fusion-able vertices. Each term is a Bray-Curtis dissimilarity estimator based on the spatial position or the size of cells. If necessary, it can be completed with other criteria; shape, dynamic... 


\subsection{Best Candidate Function}

This function gives the best vertex fusion-able to a given vertex when it exists. We propose here the following expression:

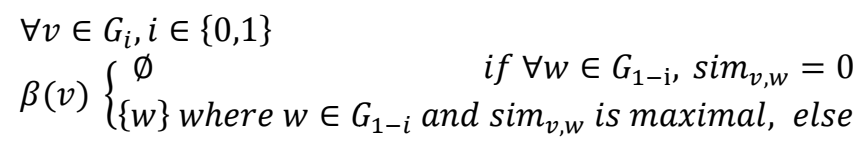

Our study case concerns successive images analysis where the overlapped areas are identical without geometrical offsets. The function $\beta$ is commutative: it is independent of the terms order and of the indexes $i$ and $j$. Reduced to the subset of vertices having a not empty image, $\beta$ becomes an involution without fixed points which allows defining the amalgamation kernel.

$$
\forall v \in G_{i}, i \in\{0,1\} \quad \mid \beta(v) \neq \varnothing \Rightarrow \beta(v) \in G_{1-i} \text { and } \beta(\beta(v))=v
$$

The key point is the area overlapping (at least one vertex satisfies the upper property). It is defined as such that bigger structure of the image is wholly overlapped. This point is developed in the result section.

\section{$5 \quad$ Results and Perspective}

Several aspects are presented to illustrate the robustness of the amalgamating method. Points in the study are discussed.

Amalgamation of different adjacency graphs of mosaic images allows tracking the radial lines over large areas of observation, as shown on two rings in (Fig. 5).

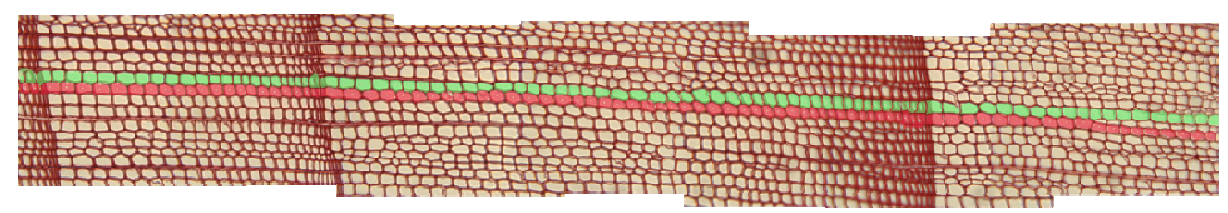

Fig. 5. Cell files through the mosaic shown in Fig 1

Transition between two successive rings is characterized by a cells morphological rupture. Note that this rupture does not affect the graphs amalgamation process, but the file tracking in image. The approach is stable regarding semi global cell property evolution.

\subsection{Influence of Blur on the Constitution of Cell Files}

Several studies have been initiated to check the blur independence of watershed which is the origin of adjacency graphs. Experiment consisted in comparing the lines of crest and the cells area on an image progressively blurred.

No significant gap is observed, illustrating good correlation between cell areas (Fig. 6. Left). The colored lines illustrate the well-known over-segment linked to the 
watershed algorithm. The Waterfall method [20] could contribute to reduce the observed differences. This method uses the result of the watershed transform on gradients of the original image to produce a sequence of images by merging some adjacent regions. Otherwise, the compared fiftieth areas are well adjusted (Fig. 6 right): the coefficient of determination and the slope of linear line tend towards 1.
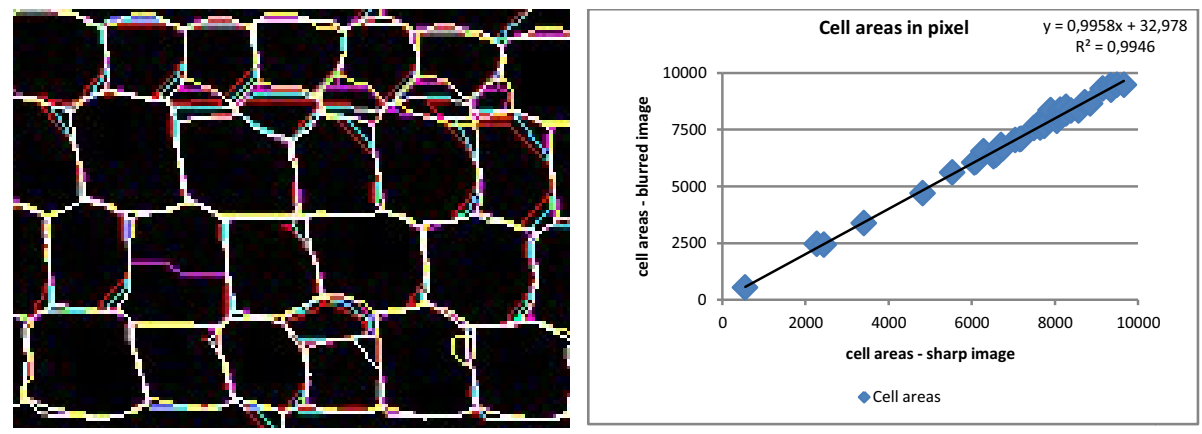

Fig. 6. Left: superposition of crest lines produced by a watershed applied to a gradually image blurred: crest lines are displayed in different color, and colors are added in case of superposition; are thus displayed in white of the image points common to all crest lines; differences are due to the over-segmentation. Right: comparison of cell area a gradually image blurred. The variation is smaller when the cell surface is higher.

\subsection{Fusion Function}

The fusion method is nearly independent from a covering rate and a blur value in the mosaic images result process (Fig. 7). Invariance rises from the robustness of the amalgamating function using only geometric criteria, insensitive to blur.

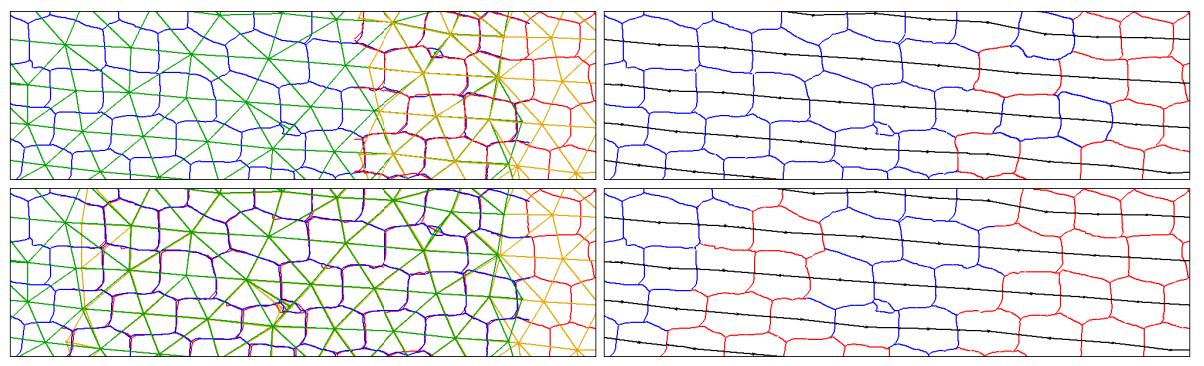

Fig. 7. Left, different overlapping of two same distinct graphs. Right, the result of two fused graphs, the cell lines are displayed in black. Bottom, with a high overlapping the results are essentially identical: difference comes from cell chosen (the least blurred).

We merge two cells by choosing one of the vertices of the fusion kernel (see previous $\S$ ), and more specifically the less blurred cell. The local blur estimator, based on the relationship between local dynamics and intensity differences [21], is used to finalize the choice. In addition, knowledge of stage displacement is an advantage, 
especially to identify the potentially cells at merging, i.e. close enough. We are currently studying the possibility of replacing in the function fusion the position terms by a term of the dynamics of cell: this point requires characterizing the local blur in image to normalize the signature of each cell. Such an indicator is presented in the definition of reliability coefficients [22] and used to validate the produced quantitative results. Use this coefficient to correct measurements is seriously considered, particularly to homogenize the intensities cells signing.

\subsection{Overlapping of Images}

The overlapping rate of images depends on the size of the bigger structure of the image. The gymnosperms (Fig. 5.) are composed of very regular cells, with weak size variation. It is easy to fix the optimal overlap during the images shooting so that at least one cell is wholly in overlapping area. The overlapping value is experimentally set to thirty micrometers, i.e. close to the three biggest cell sizes. This gives a safety margin to process the disturbed biological configurations. On the other hand, the Angiosperms (Fig. 8) present very different sizes of structures: set the overlapping size is difficult and is actually left to the biologist. Here, the overlapping width is $400 \mathrm{mi}-$ crometers so that the bigger cell is wholly overlapped.

These big cells, called vessels, cause progressive or brutal cell deformations. Images contain cells with size and shape strongly variable. It is usual to find vessels 10 to 20 times larger than fibers.

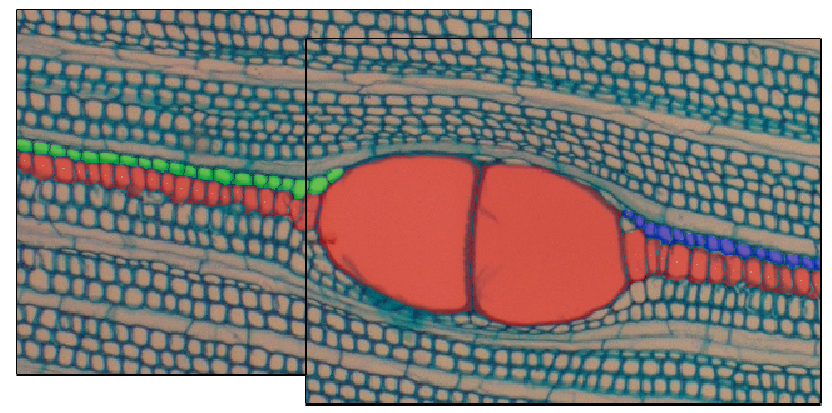

Fig. 8. Case of high structure geometry changes: graphs fusion still works when the biggest structure is totally recovered

In Fig. 8, the central file (in red) is properly assembled, thanks to the full vessel structure coverage. Squashing of fibers produced by vessel development causes discontinuities in peripheral files (in green and blue), discontinuities that fusion method cannot correct. It is necessary to implement a method of files concatenation from study of files relationship. Complementary work will soon be engaged to automatically set the optimal covering by studying the distribution of cell sizes. Study will seek a solution for cases where the covering of vessels can only be partial. 


\section{Conclusion}

Image mosaic processing is a classical research thread in vision. We presented a generic method for aggregating results by merging disjoint single-graphs, i.e. undirected graphs without loops; as an alternative to image stitching.

We defined a fusion function of vertices based on the evaluation of a similarity coefficient. This function is an involution without fixed point allowing the determining of the amalgamating kernel, i.e. the set of points that are two by two merged. The similarity coefficient does not depend on the graph structure, but only on attributes contained by vertices of the graphs. So it is adaptable to other attributes in regard to the study object.

We have developed and implemented this method in the context of tracking cell files in image mosaics describing large viewing zones. Cells individualization in each image was obtained by watershed algorithm; the obtained crests lines are used to define the cells adjacency graph, which allows to identify cell files. It is defined as the straightest paths whose adjacent nodes have similar attributes. Aggregation of files on consecutive images in the mosaic is realized by fusing graphs in order to avoid the inconvenience related to the stitching: radiometric equalization, image repositioning, blur correction and local deformation compensation...

Applying the method requires only to define explicitly the similarity coefficient and involution. The similarity function is defined by a product of normalized Brayand-Curtis dissimilarity estimators describing the geometrical aspects -position and size- of cells which can potentially be merged. In case of merging, only the less blurred cell is retained and the local blur estimator is based on the relationship between local dynamics and intensity differences. A study is engaged to introduce a component characterizing the dynamics of the cell, and so to be free of positioning criteria. Finally, the fusion method is insensitive to image blurring.

This generic method could be applied on other kinds of images of very high definition when we wish to avoid stitching as for example satellite images.

Acknowledgment. The authors gratefully acknowledge Yves CARAGLIO and Christine HEINZ for their involvement in this work, their advices and availability. A big thank to Michael GUEROULT without whom the laboratory work would not have been possible.

This work is jointly funded by a doctoral fellowship of the Labex NUMEV, by the SIBAGHE Graduate School of the University Montpellier 2 and by the Scientific Council of the University Montpellier 2.

\section{References}

1. Lézoray, O., Grady, L.: Image processing and Analysis With Graphs. CRC Press (2012)

2. Cousty, J., Bertrand, G., Couprie, M., Najman, L.: Fusion graphs: merging properties and watersheds. Journal of Mathematical Imaging and Vision 30, 87-104 (2008) 
3. Baldevbhai, P.J., Anand, R.S.: Review of graph, medical and color Image base Segmentation Techniques. Journal of Electronical and Electronics Engineering 1, 1-19 (2012)

4. Peng, B., Zhang, L., Zhang, D., Yang, J.: Image segmentation by iterated region merging with localized graph cuts. Pattern Recognition 44, 10-11 (2013)

5. Brown, M., Lowe, D.G.: Automatic Panoramic Image Stitching using Invariant Features. International Journal of Computer Vision 74, 59-73 (2007)

6. Lowe, D.G.: Distinctive image features from scale-invariant keypoints. International Journal of Computer Vision 60, 91-110 (2004)

7. Clenti, C.: Architectures flot de données dédiées au traitement d'images par Morphologie Mathématique, Thèse de doctorat en morphologie mathématique. In: ENSMP,vol. (431) (2009)

8. Sun, C., Beare, R., Hilsenstein, V., Jackway, P.: mosaicing of microscope images with global geometric and radiometric corrections. Journal of microscopy 224, 158-165 (2006)

9. Vercauteren, T., Perchant, A., Malandain, G., Pennec, X., Ayache, N.: Robust mosaicing with correction of motions distorsions and tissue deformations for In Vivo Fibered Microscopy. Medical Image Analysis 10, 673-692 (2006)

10. Thévenaz, P., Unser, M.: User-friendly Semiautomated Assembly of Accurate Image Mosaics in Microscopy. Microscopy Research And Technique 70, 135-146 (2007)

11. Liang, C., Filion, L., Cournoyer, L.: Wood structure of biotically and climatically induced light rings in eastern larch (Larix laricina). Canadian Journal of Forest Research 27, 1538-1547 (1997)

12. Wilson, B.F.: The Growing Tree. The University of Massachusetts Press, Amherst (1970)

13. Gindl, W.: Cell-wall lignin content related to tracheid dimensions in drought-sensitive austrian pine (pinus nigra). Iawa Journal 22, 113-120 (2001)

14. Brunel, G., Borianne, P., Subsol, G., Jaeger, M., Caraglio, Y.: Automatic characterization of the cell organization in light microscopic images of wood: application to the identification of the cell files. In: Plant Growth Modeling, Simulation, Visualization and Applications, vol. 4, pp. 58-65. IEEE Press (2012) ISBN 978-1-4673-0070-4

15. Kennel, P., Subsol, G., Guéroult, M., Borianne, P.: Automatic identification of cell files in light microscopic images of conifer wood. In: 2nd International Conference on Image Processing Theory Tools and Applications, pp. 98-103 (2010)

16. Sjodahl, M., Oreb, B.: Stitching interferometric measurement data for inspection of large optical components. Optical Engineering 41, 403-408 (2002)

17. Wyant, J.C., Schmit, J.: Large field of view, high spatial resolution, surface measurements. In: International Conference on Metrology and Properties of Engineering Surfaces, vol. 38, pp. 691-698 (1998)

18. Vincent, L., Soille, P.: Watersheds in Digital Spaces: An Efficient Algorithm Based on Immersion Simulations. IEE Transactions on Pattern Analysis and Machine Intelligence 13, 583-598 (1991)

19. Tremeau, A., Colantoni, P.: Regions adjacency graph applied to color image segmentation. IEEE Transactions on Image Processing 9, 735-744 (2000)

20. Beucher, S.: Watershed, Hierarchical Segmentation and Waterfall Algorithm. In: Proc. Mathematical Morphology and its Applications to Image Processing, pp. 69-76 (1994)

21. Ladjal, S.: Blur estimation in Natural Images. In: 15e congrès francophone AFRIF-AFIA Reconnaissance des Formes et Intelligence Artificielle, pp. 112-124 (2006)

22. Brunel, G., Borianne, P., Subsol, G., Jaeger, M., Caraglio, Y.: Results reliability of the automated identification of cell files in microscopic images of gymnosperms. In: 7th International Conference on Fuctional-Structural Plant Models (2013) (in submission) 